\section{Research Square}

Preprints are preliminary reports that have not undergone peer review.

They should not be considered conclusive, used to inform clinical practice, or referenced by the media as validated information.

\title{
Evaluation of automated molecular tests for detection of SARS-CoV-2 in pooled nasopharyngeal and saliva specimens
}

\section{Hamad Al-Hail}

Sidra Medical and Research Center

Faheem Mirza

Sidra Medical and Research Center

Alaa Al Hashemi

Sidra Medical and Research Center

Muneera Naseer Ahmad

Sidra Medical and Research Center

Patrick Tang

Sidra Medical and Research Center

Mohammad Rubayet Hasan ( $\square$ mhasan@sidra.org )

Sidra Medical and Research Center https://orcid.org/0000-0002-4658-7949

\section{Short report}

Keywords: COVID-19, SARS-CoV-2, Viral RNA, RNA extraction, RT-qPCR

Posted Date: January 7th, 2021

DOI: https://doi.org/10.21203/rs.3.rs-38634/v2

License: (c) (i) This work is licensed under a Creative Commons Attribution 4.0 International License. Read Full License 


\section{Abstract}

Pooling of samples for SARS-CoV-2 testing in low prevalence settings has been used as an effective strategy to expand testing capacity and mitigate challenges associated with the shortage of supplies. We evaluated two automated molecular test systems for detection of SARS-CoV-2 RNA in pooled specimens. Pooled nasopharyngeal and saliva specimens were tested by Qiagen QIAstat-Dx Respiratory SARS-CoV-2 Panel (QIAstat) or Cepheid Xpert Xpress SARS-CoV-2 (Xpert), and the results were compared to that of standard RT-qPCR tests without pooling. In nasopharyngeal specimens, the sensitivity/specificity of the pool-testing approach, with 5 and 10 specimens per pool, were $77 \% / 100 \%(n=105)$ and $74.1 \% / 100 \%$ ( $n=260)$ by QIAstat, and $97.1 \% / 100 \%(n=250)$ and $100 \% / 99.5 \%(n=200)$ by Xpert, respectively. Pool-testing of saliva (10 specimens per pool; $n=150)$ by Xpert resulted in $87.5 \%$ sensitivity and $99.3 \%$ specificity compared to individual tests. Pool size of 5 or 10 specimens did not significantly affect the difference of RT-qPCR cycle threshold $\left(\mathrm{C}_{\mathrm{T}}\right)$ values from standard testing. RT-qPCR $\mathrm{C}_{\mathrm{T}}$ values obtained with pool testing by both QIAstat or Xpert were positively correlated with that of individual testing (Pearson correlation coefficient $r=0.85$ to $0.99, p<0.05$ ). However, the $\mathrm{C}_{\mathrm{T}}$ values from Xpert were significantly stronger $(p<0.01$, paired $\mathrm{T}$-test) than that of QIAstat in a subset of SARS-CoV-2 positive specimens, with mean differences of $-4.3 \pm 2.43$ and $-4.6 \pm 2$ for individual and pooled tests, respectively. Our results suggest that Xpert SARS-CoV-2 can be utilized for pooled sample testing for COVID-19 screening in low prevalence settings providing significant cost savings and improving throughput without affecting test quality.

\section{Introduction}

Rapid testing to detect individuals infected with Severe Acute Respiratory Syndrome coronavirus 2 (SARS-CoV-2) is central to the management of the ongoing pandemic of coronavirus disease 2019 (COVID-19) [1]. Since the beginning of the outbreak, detection of viral RNA in nasopharyngeal (NP) swab specimens by real-time reverse transcription PCR (RT-qPCR) remains the main approach for identifying patients with acute infections [2]. The choice of a method for SARSCoV-2 RT-qPCR depends on multiple factors including required test throughput, rate of positivity and the availability of resources. Pre-designed assays recommended by the World Health Organization (WHO), targeting several SARS-CoV-2 genes, were made public to enable the development of relatively inexpensive, laboratory-developed RT-qPCR tests early in the outbreak. Many commercial assays have also been developed in singleplex or multiplex formats to test for SARS-CoV-2 RNA. These tests are designed for high-complexity laboratories that perform large volume testing. However, these tests may be difficult to implement in laboratories with limited expertise in molecular testing. To this end, molecular testing devices that integrate RNA extraction and RT-qPCR with random-access features with sample-to-result capability are ideally suited for laboratories that may not be able to deal with high-complexity molecular testing. These test methods and platforms are also suitable for testing in a near point-of-care (POC) setting [3].

QIAstat-Dx Respiratory SARS-CoV-2 Panel (QIAstat) and Cepheid Xpert Xpress SARS-CoV-2 (Xpert) tests are among the few fully automated, near POC solutions for COVID-19 testing that are FDA approved under emergency use authorization (EUA). While Xpert detects multiple targets within the SARS-CoV-2 genome, QIAstat is a multiplexed RT-qPCR test for the detection of multiple respiratory pathogens, including the SARS-CoV-2 virus in nasopharyngeal specimens [4-6]. Although these tests are rapid and convenient, they are more expensive than laboratory-developed assays and have lower throughput as each instrument module can only test one sample at a time. We hypothesized that pooling of multiple specimens, in low prevalence settings, may significantly reduce the cost of the commercial, automated molecular tests and improve the throughput of these assays. Furthermore, in the face of massive surges in demand and shortages of test reagents and kits [7, 8], pooling of samples for COVID-19 testing will help laboratories perform a larger number of tests with limited test kits.

Pooling of specimens to reduce cost and expand the capacity of COVID-19 testing has been well described in the literature [9-12]. Pooled sample testing was also encouraged by FDA for the screening of asymptomatic individuals for COVID-19 [13]. While the pooled sample testing approach was evaluated using laboratory-developed, standard RT-qPCR tests, QIAstat and Xpert tests have not been evaluated for pool testing. In this study, we assessed whether pooling of nasopharyngeal and saliva specimens affects the performance characteristics of these tests. Pooled test results were verified by individual tests by a WHO recommended, standard RT-qPCR assay. For a subset of specimens, pool results were also compared to individual test results by the respective commercial tests.

\section{Material And Methods}

Evaluation of Xpert and QIAstat was performed in the Molecular Infectious Diseases Laboratory of Sidra Medicine, a 400-bed women's and children's tertiary care hospital in Qatar, which was designated as a COVID-19-free facility, as part of an integrated, national pandemic management plan. Active screening of patients for COVID-19 was started in March 05, 2020 and the rate of PCR positivity for SARS-CoV-2 has remained $<3 \%$ of the submitted samples. Standard COVID-19 testing in our laboratory involves extraction of viral RNA from nasopharyngeal flocked swab (NPFS)(BD) or saliva specimens in an automated nucleic acid extraction platform NucliSENS EasyMAG (bioMerieux) followed by RT-qPCR, based on one of the assays recommended by the WHO [14]. The performance standards of the standard method were established in our laboratory according to College of American Pathologists (CAP) guidelines. Both QIAstat and Xpert are fully automated, multiplex real-time RT-PCR tests. While QIAstat is intended to detect multiple respiratory pathogens, including SARSCoV-2, Xpert is designed to detect the E and N genes of SARS-CoV-2. A total of 815 NPFS and 150 saliva specimens were simultaneously assessed by the standard approach and one of the pool testing approaches in this study. For pool testing, $0.1 \mathrm{ml}$ of each of the 5 or 10 specimens were pooled together, vortexed for $10 \mathrm{sec}$ and $0.3 \mathrm{ml}$ of pooled specimen was analyzed by Xpert or QIAstat according to manufacturer's instructions. For a subset of samples $(n=60)$, pool test results were also verified by individual tests using respective commercial assays.

To compare SARS-CoV-2 RT-qPCR $\mathrm{C}_{\mathrm{T}}$ values from different assays, only the $\mathrm{C}_{\mathrm{T}}$ values for the $\mathrm{E}$ gene were compared. The linear correlation of RT-qPCR $\mathrm{C}_{\mathrm{T}}$ values obtained by pool testing and individual testing were evaluated by measuring Pearson correlation coefficient ( $r$ ) and associated $p$-values. The statistical significance of differences in $\mathrm{C}_{\mathrm{T}}$ values between pool-testing by QIAstat or Xpert and individual standard testing were calculated by the Mann-Whitney $\mathrm{U}$ test. The statistical significance of differences in $\mathrm{C}_{\mathrm{T}}$ values on the same specimens tested by individual and pool testing approaches by QIAstat and $\mathrm{Xpert}$ were 
calculated by paired, Student's T-test. All statistical analyzes were performed in GraphPad Prism 9.0.0. The study involves laboratory validation of test methods and the secondary use of anonymous, residual pathological specimens that falls under the category 'exempted' by the Sidra Medicine Institutional Review Board.

\section{Results}

\section{Pool testing of nasopharyngeal specimens by QIAstat for detection of SARS-CoV-2}

NPFS specimens were tested by QIAstat either in a pool of 5 specimens (pool-5) or in a pool of 10 specimens (pool-10) (Table 1). For pool-5 testing, a total of 10 pooled runs ( $n=50$ specimens) that gave positive results and 11 pooled runs ( $n=55$ specimens) that gave negative results by QIAstat were individually assessed by standard RT-qPCR. For pool-10 testing, specimens in 14 positive pools ( $n=140$ specimens) and 12 negative pools ( $n=120$ specimens) by QIAstat were individually assessed. QIAstat test results for respiratory pathogens other than SARS-CoV-2 were disregarded. By individual testing, a total of 6 pools had $>1$ positive samples in the pool-10 group ( 1 table). Among these pools, two were undetectable by QIAstat pool-10 testing. In both pool groups, all of the positive pool results were correct, but 3 of 10 negative pool- 5 results and 5 of 12 negative pools in pool- 10 results were falsely negative after individual assessment. The sensitivity, specificity and accuracy of QIAstat pool-5 and pool-10 against standard individual testing were not significantly different (Table 1). A total of 10 positive results were missed by the QIAstat pool testing approach: the RT-qPCR $C_{T}$ values by the standard method for these specimens ranged from 32.6 to 38.3. To investigate whether lower analytical sensitivity of QIAstat was responsible for these discrepant results, we individually tested some of these specimens by QIAstat and noted that the positive samples, that were missed by pooled testing, were also negative by individual QIAstat testing, although they were positive by two other methods ( 2 table). The mean $\mathrm{C}_{\mathrm{T}}$ values for these samples by standard RT-qPCR and Xpert were $35.1 \pm 1.5$ and $35.1 \pm 1.9$ respectively. For samples that were positive by both pool testing and individual testing approach, the RT-qPCR $\mathrm{C}_{\mathrm{T}}$ values were positively correlated (Pearson correlation coefficient $\mathrm{r}=0.9719$ and $0.9181 ; p<0.001$; for pool- 5 and pool- 10 , respectively) (Figs $1 \mathrm{~A}$ and $1 \mathrm{~B})$. Mean $\mathrm{C}_{\mathrm{T}}$ value change $\left(\mathrm{DC}_{\mathrm{T}}\right)$ because of pooling 5 and 10 specimens were not significantly different from each other $(p=0.5354)$ (Fig 1F).

\section{Pool testing of nasopharyngeal specimens by Xpert for detection of SARS-CoV-2}

For pool-5 testing of NP swabs by Xpert, a total of 31 pooled runs ( $\mathrm{n}=155$ specimens) that gave positive results and 19 pooled runs ( $\mathrm{n}=95$ specimens) that gave negative results by Xpert were individually assessed by standard RT-qPCR. For pool- 10 testing, specimens in 11 positive pools ( $\mathrm{n}=110$ specimens) and 9 negative pools ( $n=90$ specimens) by Xpert were individually assessed. By individual testing, a total of 4 pools had $>1$ positive samples in the pool- 5 group ( $\mathrm{S} 1$ table). All pool test results, except one, were correct in each of the pool-5 and pool-10 groups. The sensitivity, specificity and accuracy of Xpert pool testing in NP specimens against standard, individual testing were $>95 \%$ in both group and were not affected by pool size of 5 or 10 specimens (Table 1 ). The samples that gave a false negative result in pool- 5 had a PCR $\mathrm{C}_{\mathrm{T}}$ value of 39 by the standard test, and the sample that gave a false positive result in pool-10 had a PCR $\mathrm{C}_{\mathrm{T}}$ value of 42.5 by Xpert. For samples that were positive by both methods, the RT-qPCR $\mathrm{C}_{\mathrm{T}}$ values were positively correlated (Pearson correlation coefficient $\mathrm{r}=$ 0.9719 and 0.9583 ; $p<0.0001$; for pool-5 and pool-10, respectively) (Fig $1 \mathrm{C}$ and $1 \mathrm{D}$ ). Mean $\mathrm{C}_{\mathrm{T}}$ value change ( $\mathrm{DC}_{\mathrm{T}}$ ) because of pooling 5 and 10 specimens were not significantly different from each other $(p=0.4765)$ (Fig $1 F)$. However, the mean $\mathrm{C}_{\mathrm{T}}$ value difference of QIAstat vs Xpert pool testing $\left(\mathrm{DC} \mathrm{C}_{\mathrm{T}}\right)$ from standard testing were significantly different $(\mathrm{p}<0.01)($ Fig $1 F)$.

\section{Pool testing of saliva specimens by Xpert for detection of SARS-CoV-2}

For testing of pooled saliva specimens by Xpert a total of 8 pooled runs ( $n=80$ specimens) that gave positive results and 7 pooled runs $(n=70$ specimens) that gave negative results were individually assessed by standard RT-qPCR. All except two (87 \%) pooled test results were correct. The sensitivity, specificity and accuracy of saliva pool testing by Xpert against standard, individual testing were $87.5 \%, 99.3 \%$ and $98.7 \%$, respectively (Table 1 ). For samples that were positive by both methods, the RT-qPCR $\mathrm{C}_{\mathrm{T}}$ values were positively correlated (Pearson correlation coefficient $\left.\mathrm{r}=0.8535, p<0.05\right)(\mathrm{Fig} 1 \mathrm{E})$. Mean $\mathrm{C}_{\mathrm{T}}$ value change $\left(D_{\mathrm{T}}\right)$ because of pooling 10 saliva specimens was not significantly different from pool-10 testing of NP swab specimens ( $\left.p=0.6507\right)$ (Fig $1 \mathrm{~F}$ ).

\section{Correlation and comparison of $\mathrm{C}_{\mathrm{T}}$ values by QIAstat and Xpert tests in individual and pooled specimens}

For a direct comparison of $C_{T}$ values, we tested a subset of known positive specimens $\left(n=6 ; C_{T}<30\right.$ by standard testing) by both QIAstat and Xpert tests both individually and in a pool of 10 NPFS specimens. The $\mathrm{C}_{\mathrm{T}}$ values obtained by individual versus pool-testing were positively correlated by both QIAstat $(r=0.9868, p<0.001)$ and Xpert $(r=8631, p<0.05)$ testing (Fig 2A and $B)$. The mean $C_{T}$ value changes $\left(D_{T}\right)$ because of pooling 10 NPFS specimens were -3.683 $(95 \% \mathrm{Cl},-5.106$ to -2.261$)$ and $-3.333(95 \% \mathrm{Cl},-5.861$ to -0.8054$)$ by QIAstat and Xpert, respectively, which was not significantly different from each other $(p=0.7316)$ (Fig 2C). However, $\mathrm{C}_{\mathrm{T}}$ values obtained by Xpert were significantly ( $\mathrm{p}<0.01$ by paired, Student's T-test) lower or stronger than those of QIAstat tests, with mean difference of $-4.250(95 \% \mathrm{Cl},-6.795$ to -1.705$)$ and $-4.6(95 \% \mathrm{Cl},-6.703$ to 2.497$)$ for individual and pooled tests, respectively (Fig $2 \mathrm{D})$.

\section{Discussion}

QIAstat and Xpert tests are among the commercial rapid multiplex PCR assays for SARS-CoV-2 detection that have recently been independently evaluated and demonstrated to have high sensitivity and specificity against standard RT-qPCR tests [4-6]. These tests are easy to perform without any specific special skills and are suitable for near POC applications, requiring approximately 30-70 min in instrument run-time. However, the higher test cost makes them less suitable as a routine screening tests. The test equipment are modular, where each module can process only one specimen at a time. In order to improve the throughput of these assay, large number of modules are necessary which requires a larger capital investment. As an alternative solution, we performed pooled specimen testing using these platforms and compared the accuracy of results with that of standard RT-qPCR. Our new pooled test approach demonstrated equivalent results to standard testing, in particular with Xpert test and significantly saved costs. The lower sensitivity of pooled sample run using QIAstat is related to the 
lower analytical sensitivity of the assay compared to other methods (Fig 2, S1 table) rather than because of sample pooling. For both test platforms, pool size of 5 or 10 specimens did not significantly affect the sensitivity of the pooled testing approach (Table 1, Fig 1F). While pool-10 testing may provide more costsavings than pool-5 testing, smaller pool size may help reducing wait time for specimens in low-volume testing laboratories and thus improve the turnaround time. In our setting, after implementation of pooled testing with pool-sizes of 3- to 10-specimens per test, based on specific cut-off times for specimenreceiving, $<10 \%$ of pooled samples required re-testing. However, in order to avoid re-testing of large numbers of samples, pooled testing should only be implemented in low-prevalence settings and the size of the pools must be adjusted to match the current prevalence in the test population.

With the continued rise in COVID-19 cases worldwide and urgent need for easing up lockdown and social distancing measures for economic reasons, the role of COVID-19 screening in asymptomatic individuals has become more important than ever. Therefore, there is a heightened interest in inexpensive and convenient test methods for large volume testing and decentralization of the testing process. Sample pooling for COVID-19 testing has now been recognized by WHO, CDC and FDA $[13,15,16]$. Automated molecular test platforms such as Cepheid GeneXpert systems are easy to be implemented in any setting and pooled sample tests in these platforms can reduce the cost by 5 to 10 -fold and bring down the per test cost equivalent to laboratory-developed tests. FDA recommends that pooled tests be in $\geq 85 \%$ percent positive agreement with individual tests. Our results show that Xpert tests can be successfully applied for pooled testing of up to 10 -specimens with equivalent clinical sensitivity to that of standard, individual testing, significantly reduce cost and improve the test capacity of new and existing test facilities.

\section{Conclusions}

In conclusion, we evaluated a modified approach for COVID-19 testing by QRSP assay. We demonstrate that pooling of up to 10 specimens does not significantly affect the sensitivity of the assay, compared to standard RT-qPCR, but improve the TAT of negative test reporting. In order to save cost, and improve throughput and TAT of negative test results, pooled QRSP testing approach can be utilized for COVID-19 screening in low prevalence setting, in particular for screening in COVID-19-free hospitals or in health care facilities in countries where the number of cases are low or declining.

\section{Declarations}

\section{Ethics approval and consent to participate:}

The study involves laboratory validation of test methods and the secondary use of anonymous, residual pathological specimens that falls under the category 'exempted' by Sidra Medicine Institutional Review Board.

\section{Consent for publication:}

Not applicable. The manuscript does not contain any individual person's data in any form (including individual details, images or videos).

\section{Availability of data and materials:}

All data generated or analyzed during this study are included in this published article.

\section{Competing interests:}

The authors declare that they have no competing interests.

\section{Funding:}

Not applicable.

\section{Authors' contributions:}

$\mathrm{MRH}$ analyzed data and wrote the manuscript; $\mathrm{MRH}$ and $\mathrm{HAH}$ designed the study; $\mathrm{HAH}, \mathrm{FM}, \mathrm{AAH}$ and MNA performed laboratory work; PT provided valuable suggestions and revised the manuscript.

\section{Acknowledgements:}

We are grateful for the efforts of all technologists in the Molecular Infectious Disease Laboratory at Sidra Medicine.

\section{References}

1. Zhai P, Ding Y, Wu X, Long J, Zhong Y, Li Y. The epidemiology, diagnosis and treatment of COVID-19. Int J Antimicrob Agents. 2020;55(5):105955. Epub 2020/04/03. doi: 10.1016/j.jjantimicag.2020.105955. PubMed PMID: 32234468; PubMed Central PMCID: PMCPMC7138178.

2. CDC. Information for Laboratories about Coronavirus (COVID-19) 2020 [cited 2020 June 12, 2020]. Available from: https://www.cdc.gov/coronavirus/2019-nCoV/lab/index.html.

3. Tang YW, Schmitz JE, Persing DH, Stratton CW. Laboratory Diagnosis of COVID-19: Current Issues and Challenges. J Clin Microbiol. 2020;58(6). Epub 2020/04/05. doi: 10.1128/JCM.00512-20. PubMed PMID: 32245835; PubMed Central PMCID: PMCPMC7269383.

4. Visseaux B, Le Hingrat Q, Collin G, Bouzid D, Lebourgeois S, Le Pluart D, et al. Evaluation of the QIAstat-Dx Respiratory SARS-CoV-2 Panel, the first rapid multiplex PCR commercial assay for SARS-CoV-2 detection. J Clin Microbiol. 2020. Epub 2020/04/29. doi: 10.1128/JCM.00630-20. PubMed PMID: 
32341142

5. Zhen W, Smith E, Manji R, Schron D, Berry GJ. Clinical Evaluation of Three Sample-to-Answer Platforms for Detection of SARS-CoV-2. J Clin Microbiol. 2020;58(8). Epub 2020/04/26. doi: 10.1128/JCM.00783-20. PubMed PMID: 32332061; PubMed Central PMCID: PMCPMC7383520.

6. Loeffelholz MJ, Alland D, Butler-Wu SM, Pandey U, Perno CF, Nava A, et al. Multicenter Evaluation of the Cepheid Xpert Xpress SARS-CoV-2 Test. J Clin Microbiol. 2020;58(8). Epub 2020/05/06. doi: 10.1128/JCM.00926-20. PubMed PMID: 32366669; PubMed Central PMCID: PMCPMC7383535.

7. ASM. Supply shortages impacting COVID-19 and non-COVID testing: ASM press; 2020 [cited 2020 October 20]. Available from: https://asm.org/Articles/2020/September/Clinical-Microbiology-Supply-Shortage-Collecti-1.

8. ASM. Laboratory Supply Shortages Are Impacting COVID-19 and Non-COVID Diagnostic Testing 2020 [cited 2020 October 20]. Available from: https://asm.org/Articles/2020/September/Laboratory-Supply-Shortages-Are-Impacting-COVID-19? utm_source=RealMagnet\&utm_medium=email\&utm_content=COVID\%2D19\%5FNews\%5F20201021\&utm_campaign=Sci\%20Topics\%20\%3E\%20COVID\%2

9. Lim KL, Johari NA, Wong ST, Khaw LT, Tan BK, Chan KK, et al. A novel strategy for community screening of SARS-CoV-2 (COVID-19): Sample pooling method. PLoS One. 2020;15(8):e0238417. Epub 2020/08/29. doi: 10.1371/journal.pone.0238417. PubMed PMID: 32857823; PubMed Central PMCID: PMCPMC7454965.

10. Fogarty A, Joseph A, Shaw D. Pooled saliva samples for COVID-19 surveillance programme. Lancet Respir Med. 2020. Epub 2020/09/26. doi: 10.1016/S2213-2600(20)30444-6. PubMed PMID: 32976755; PubMed Central PMCID: PMCPMC7508511.

11. Lohse S, Pfuhl T, Berko-Gottel B, Rissland J, Geissler T, Gartner B, et al. Pooling of samples for testing for SARS-CoV-2 in asymptomatic people. Lancet Infect Dis. 2020. Epub 2020/06/13. doi: 10.1016/S1473-3099(20)30362-5. PubMed PMID: 32530425; PubMed Central PMCID: PMCPMC7194818.

12. Yelin I, Aharony N, Shaer Tamar E, Argoetti A, Messer E, Berenbaum D, et al. Evaluation of COVID-19 RT-qPCR test in multi-sample pools. Clin Infect Dis. 2020. Epub 2020/05/03. doi: 10.1093/cid/ciaa531. PubMed PMID: 32358960; PubMed Central PMCID: PMCPMC7197588.

13. FDA. Pooled Sample Testing and Screening Testing for COVID-19 2020 [cited 2020 October 20]. Available from: https://www.fda.gov/medicaldevices/coronavirus-covid-19-and-medical-devices/pooled-sample-testing-and-screening-testing-covid-19\#pooled.

14. Corman VM, Landt O, Kaiser M, Molenkamp R, Meijer A, Chu DK, et al. Detection of 2019 novel coronavirus (2019-nCoV) by real-time RT-PCR. Euro Surveill. 2020;25(3). Epub 2020/01/30. doi: 10.2807/1560-7917.ES.2020.25.3.2000045. PubMed PMID: 31992387; PubMed Central PMCID: PMCPMC6988269.

15. WHO. Pooling samples boosts Ghana's COVID-19 testing 2020 [cited 2020 October 20]. Available from: https://www.who.int/news-room/featurestories/detail/pooling-samples-boosts-ghana-s-covid-19-testing.

16. CDC. Interim Guidance for Use of Pooling Procedures in SARS-CoV-2 Diagnostic, Screening, and Surveillance Testing 2020 [cited 2020 October 20 ]. Available from: https://www.cdc.gov/coronavirus/2019-ncov/lab/pooling-procedures.html.

\section{Table}

Table 1: Comparison of pooled test results with individual standard RT-qPCR test results

\begin{tabular}{|c|c|c|c|c|c|c|c|c|c|c|c|}
\hline Test (specimen) & $\begin{array}{l}\text { Pool } \\
\text { size }\end{array}$ & $\begin{array}{c}\text { No of } \\
\text { Specimen }\end{array}$ & $\begin{array}{c}\text { Positive } \\
\text { pool }\end{array}$ & $\begin{array}{c}\text { Negative } \\
\text { pool }\end{array}$ & $\begin{array}{c}\text { True } \\
\text { positive }\end{array}$ & $\begin{array}{c}\text { False } \\
\text { positive }\end{array}$ & $\begin{array}{c}\text { True } \\
\text { negative }\end{array}$ & $\begin{array}{c}\text { False } \\
\text { negative }\end{array}$ & $\begin{array}{c}\text { Sensitivity, } \\
\% \\
(95 \% \mathrm{CD})\end{array}$ & $\begin{array}{c}\text { Specificity, } \\
\% \\
(95 \% \mathrm{CI})\end{array}$ & $\begin{array}{c}\text { Accuracy, } \\
\% \\
(95 \% \mathrm{CD})\end{array}$ \\
\hline \multirow[t]{2}{*}{ QIAstat (NP swab) } & 5 & 105 & 10 & 11 & 10 & 0 & 92 & 3 & $\begin{array}{c}77.0 \\
(46.9-95.0)\end{array}$ & $\begin{array}{c}100 \\
(96.1-100)\end{array}$ & $\begin{array}{c}96.3 \\
(91.9-99.4)\end{array}$ \\
\hline & 10 & 260 & 14 & 12 & 20 & 0 & 233 & 7 & $\begin{array}{c}74.1 \\
(54.7-88.9)\end{array}$ & $\begin{array}{c}100 \\
(98.4-100)\end{array}$ & $\begin{array}{c}97.3 \\
(94.5-98.9)\end{array}$ \\
\hline \multirow[t]{2}{*}{$\begin{array}{l}\text { Cepheid (NP } \\
\text { swab) }\end{array}$} & 5 & 250 & 31 & 19 & 34 & 0 & 215 & 1 & $\begin{array}{c}97.1 \\
(85.1-99.9)\end{array}$ & $\begin{array}{c}100 \\
(98.1-100)\end{array}$ & $\begin{array}{c}99.6 \\
(99.5-99.9)\end{array}$ \\
\hline & 10 & 200 & 11 & 9 & 10 & 1 & 189 & 0 & $\begin{array}{c}100 \\
(69.1-100)\end{array}$ & $\begin{array}{c}99.5 \\
(97.1-99.9)\end{array}$ & $\begin{array}{c}99.5 \\
(97.3-99.9)\end{array}$ \\
\hline Cepheid (Saliva) & 10 & 150 & 8 & 7 & 7 & 1 & 141 & 1 & $\begin{array}{c}87.5 \\
(47.4-99.7)\end{array}$ & $\begin{array}{c}99.3 \\
(96.1-100)\end{array}$ & $\begin{array}{c}98.7 \\
(95.3-99.9)\end{array}$ \\
\hline
\end{tabular}

\section{Figures}


Figure 1
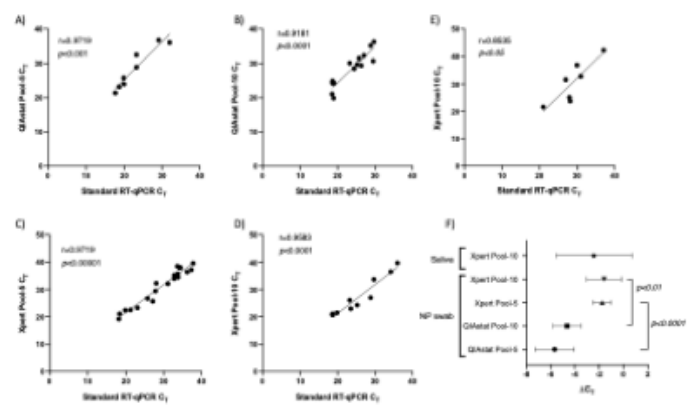

\section{Figure 1}

Correlation between RT-qPCR CT values obtained by pooled testing and standard methods. CT values obtained from QIAstat pool-5 (A) and pool-10 testing (B) and Xpert pool-5 (C) and pool-10 testing (D) in NP specimens and Xpert pool-10 testing in saliva specimens (E) were plotted against CT values obtained by standard testing and fitted in a linear regression model (Pearson correlation coefficient $=r$ ). For the positive pools that gave multiple positive results by individual assessment, the lowest (strongest) CT was used for analysis (S1 table). (F) mean CT value difference ( $\triangle \mathrm{CT}$ ) between pool CT and standard individual CT with $95 \%$ confidence intervals $(\mathrm{Cl})$. Statistical significance $(\mathrm{p})$ of difference of $\Delta \mathrm{CT}$ between different groups were calculated by Mann-Whitney U test.

Figure 2
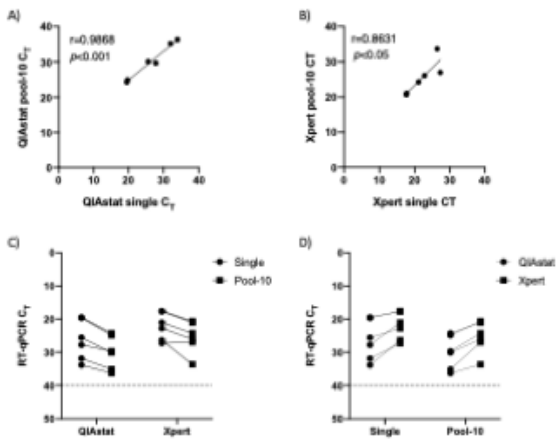

\section{Figure 2}

Correlation between CT values obtained by pooled testing and individual testing by QIAstat and Xpert tests. CT values obtained from QIAstat pool-10 (A) and Xpert pool-10 (B) were plotted against individual CT values obtained by respective methods and fitted in a linear regression model (Pearson correlation coefficient $=r)$. CT values are compared between single versus pool-10 testing by QIAstat and Xpert tests $(C)$. CT values are compared between QIAstat versus Xpert tests with individual and pool-10 testing (D).

\section{Supplementary Files}

This is a list of supplementary files associated with this preprint. Click to download.

- Supplementarymaterials.docx 